\section{Occupational Exposure to Blood or Body Fluids as a Result of Needlestick Injuries and Other Sharp Device Injuries Among Medical Residents in Japan}

TO THE EDITOR-Medical residents are vulnerable to needlestick injuries and/or injuries from other sharp devices (hereafter referred to as needlestick and/or sharps injuries) because they lack experience and skill. ${ }^{1,2}$ In the United States, $71 \%$ of medical residents and medical students reported 1 or more needlestick and/or sharps injuries or other blood or body-fluid exposures every year.'

The incidence rate of needlestick and/or sharps injury describes the proportion of people who sustained such injuries during a specified period of time-for example, during the total time in which people in the population were at risk. Incidence rate is a precise estimate of the rate at which a particular event occurs. The incidence rate of needlestick and/ or sharps injury among medical residents has been determined in some countries ${ }^{3-5}$; however, there have been no reports on the incidence rate among medical residents in Japan. The aim of this study was to determine the monthly incidence rate of needlestick and/or sharps injury among medical residents at 14 training hospitals in Japan and to compare the incidence rate among first-year medical residents with that among second-year medical residents.

A self-reporting questionnaire was mailed to 231 medical residents (127 first-year residents and 104 second-year residents) at 14 teaching hospitals throughout Japan. The questionnaire was anonymous to protect the privacy of the respondents, who were participating in a compulsory 2-year training program for various specialties, including internal medicine, surgery, pediatrics, emergency medicine, obstetrics, and gynecology.

The residency training program in which respondents were participating began in the hospitals in May. The survey was done at the end of August 2005; thus, it was assumed that the first-year residents had spent 4 months in the program and the second-year residents had spent 16 months in the program. The incidence rate of needlestick and/or sharps injury per person-month was calculated using the number of needlestick and/or sharps injuries experienced and the time that the respondents had spent in training.

All statistical analysis was performed using Statistical Package for the Social Sciences 10.0 for Windows (SPSS). The Mann-Whitney $U$ test was used to determine the difference in the incidence rate of needlestick and/or sharps injury between the first-year medical residents and the second-year medical residents.

The Human Research Committee of Kitasato University, Japan, approved this study's recruitment procedures, procedures for obtaining consent, and field procedures before the survey was conducted.

One hundred sixteen first-year medical residents and 76 second-year medical residents participated, of which 147 were men and 45 were women. The participation rate was $83.1 \%$.

TA B LE. Needlestick and/or Sharp Device Injuries Reported by Respondents at Participating Hospitals

\begin{tabular}{|c|c|c|c|c|c|}
\hline \multirow[b]{2}{*}{ Hospital } & \multirow[b]{2}{*}{ No. of beds } & \multicolumn{2}{|c|}{ No. of respondents } & \multicolumn{2}{|c|}{$\begin{array}{l}\text { No. of needlestick and } \\
\text { sharps injuries reported }\end{array}$} \\
\hline & & $\begin{array}{l}\text { First-year } \\
\text { residents }\end{array}$ & $\begin{array}{l}\text { Second-year } \\
\text { residents }\end{array}$ & $\begin{array}{l}\text { Among } \\
\text { first-year } \\
\text { residents }\end{array}$ & $\begin{array}{l}\text { Among } \\
\text { second-year } \\
\text { residents }\end{array}$ \\
\hline 1 & $200-249$ & 6 & 1 & 4 & 1 \\
\hline 2 & $300-349$ & 9 & 8 & 9 & 7 \\
\hline 3 & $300-349$ & 7 & 5 & 4 & 11 \\
\hline 4 & $300-349$ & 10 & 4 & 4 & 3 \\
\hline 5 & $300-349$ & 3 & 1 & 1 & 3 \\
\hline 6 & $300-349$ & 7 & 3 & 1 & 0 \\
\hline 7 & $300-349$ & 14 & 13 & 8 & 6 \\
\hline 8 & $300-349$ & 3 & 5 & 3 & 3 \\
\hline 9 & $300-349$ & 6 & 4 & 7 & 4 \\
\hline 10 & $350-399$ & 6 & 2 & 3 & 2 \\
\hline 11 & $350-399$ & 8 & 5 & 0 & 1 \\
\hline 12 & $350-399$ & 9 & 3 & 3 & 2 \\
\hline 13 & $400-449$ & 10 & 8 & 3 & 7 \\
\hline 14 & $400-449$ & 18 & 14 & 23 & 20 \\
\hline Overall & $\ldots$ & 116 & 76 & 73 & 70 \\
\hline
\end{tabular}




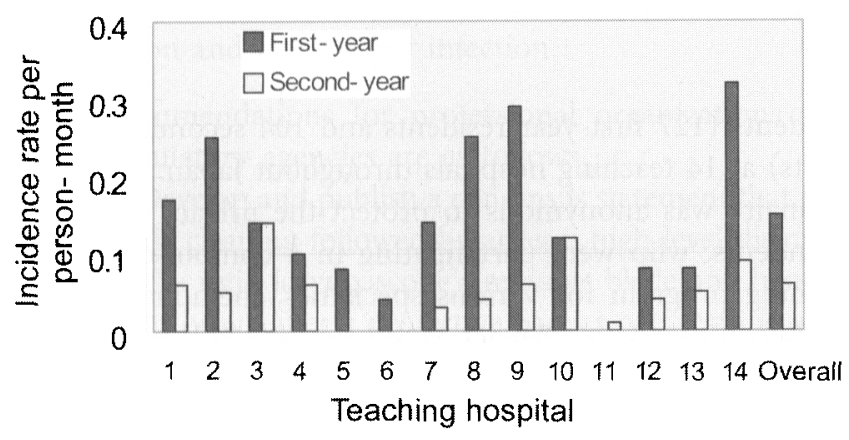

FIGURE. The incidence rate of needlestick injuries and/or sharp device injuries among first-year and second-year medical residents, by hospital.

The response rates varied among the hospitals, ranging from $53.8 \%$ to $100 \%$. The Table shows the size of the participating hospitals in terms of the number of beds; it also includes the number of respondents and the number of needlestick and/ or sharps injuries at each hospital. Among the respondents, $37(31.9 \%)$ of 116 first-year medical residents reported sustaining 73 needlestick and/or sharps injuries, and $42(55.3 \%)$ of 76 second-year medical residents reported sustaining 70 needlestick and/or sharps injuries since they started residency training. The median number of needlestick and/or sharps injuries was 0 (range, 0-4) for the first-year medical residents and 1 (range, $0-4$ ) for the second-year medical residents.

The Figure shows the incidence rates of needlestick and/ or sharps injury for the first-year and second-year medical residents at each hospital. Differences in the incidence rate among the hospitals were observed. The incidence rates were 0.15 incidents per person-month (95\% confidence interval [CI], 0.12-0.19) for the first-year medical residents and 0.06 incidents per person-month ( $95 \% \mathrm{CI}, 0.05-0.08$ ) for the second-year medical residents; this was a significant difference $(P<.05)$.

During the first 4 months of their training, approximately $32 \%$ of the first-year medical residents reported 1 or more needlestick and/or sharps injuries; approximately $55 \%$ of the second-year medical residents reported 1 or more needlestick and/or sharps injuries during their 16 months of training. The incidence rate of needlestick and/or sharps injury among the first-year medical residents was significantly higher than that among the second-year medical residents. The risk of needlestick and/or sharps injuries during the first 4 months of training was about 2.5 -fold higher than that during the 16-month training period.

The incidence rate of needlestick and/or sharps injury among medical residents in the United States is 0.11 incidents per person-month. ${ }^{3}$ In Taiwan, the incidence rate of needlestick and/or sharps injury is also 0.11 incidents per per- son-month among healthcare workers, including physicians, nurses, laboratory technicians, and cleaners." The incidence rate in the present study is similar to that observed in these studies.

Previous studies showed that there were differences in the incidence rate of needlestick and/or sharps injury among specialties. Heald et al. ${ }^{4}$ reported that the incidence rates among medical residents in the United States were 0.05 incidents per person-month among nonsurgical residents and 0.32 incidents per person-month among surgical residents. The incidence rates among surgical residents were relatively higher, ranging from 0.19 incidents per person-month ${ }^{3}$ to 0.45 incidents per person-month. ${ }^{7}$ The data obtained in this study do not include information on the specialties in which the residents worked when their injuries occurred. Because the residency training program includes surgical training, further studies are needed to identify differences in the incidence rate among Japanese medical residents being trained in various specialties.

Because the incidence rate of needlestick and/or sharps injury was lower among the second-year medical residents than among the first-year medical residents, training and education about how to prevent such injuries might reduce the incidence rate, particularly during the initial period of residency training. ${ }^{5}$ Providing education about prevention and about use of standard precautions is essential for increasing the awareness of potential risk among medical residents. ${ }^{8}$

Protection strategies, such "double gloving" during procedures with higher likelihoods of exposure and using safetyengineered medical devices, have been implemented in healthcare facilities. Installing safety-engineered devices apparently contributes to a significant decrease in needlestick and/or sharps injuries across occupation. ${ }^{9}$ Information on whether the participating hospitals implemented these protective strategies was not obtained in this study. The use of protective strategies might be associated with the differences in the incidence rates of needlestick and/or sharps injury observed among the training hospitals in this study.

There are a few limitations to this study. The information obtained was based on self-reported data; thus, medical residents might not accurately report or recall the number of actual needlestick and/or sharps injuries that occurred. However, the underreporting of needlestick and/or sharps injuries is a concern only when cases reported to the hospitals are analyzed. ${ }^{2,3,5}$ This bias could be minimized using an anonymous questionnaire and asking for information on the number of needlestick and/or sharps injuries that occurred during shorter periods of time.

Koji Wada, MD, MSc; Rie Narai, MD; Yumi Sakata, MD; Toru Yoshikawa, MD; Masashi Tsunoda, MD, PhD; Katsutoshi Tanaka, MD, PhD; Yoshiharu Aizawa, MD, PhD 
From the Department of Occupational Health (K.W.) and the Department of Occupational Mental Health (Y.S., K.T.), Graduate School of Medical Sciences, and the Department of Preventive Medicine and Public Health (M.T., Y.A.), Faculty of Medicine, Kitasato University, and the Health Promotion Centre, Mazda Motor Corporation, Hiroshima (R.N.), Sagamihara, and the Institute for Science of Labour, Kasawaki (T.Y), Kanagawa, Japan; and the Department of Epidemiology, Biostatistics, and Occupational Health, McGill University, Montreal, Quebec, Canada (K.W.).

Address reprint requests to Koji Wada, MD, MSc, 1-15-1 Kitasato Sagamihara, Kanagawa, 228-8555, Japan, Department of Occupational Health, Graduate School of Medical Sciences, Sagamihara, Kanagawa, Japan (kwada-sgy@umin.ac.jp).

Infect Control Hosp Epidemiol 2007; 28:507-509

(C) 2007 by The Society for Healthcare Epidemiology of America. All rights reserved. 0899-823X/2007/2804-0026\$15.00. DOI: $10.1086 / 518096$

\section{REFERENCES}

1. O'Neil TM, Abbott AV, Radecki SE. Risk of needlesticks and occupational exposures among residents and medical students. Arch Intern Med 1992; 152:1451-1456.

2. Resnic FS, Noerdlinger MA. Occupational exposure among medical students and house staff at a New York City Medical Center. Arch Intern Med 1995; 155:75-80.

3. Radecki S, Abbott A, Eloi L. Occupational human immunodeficiency virus exposure among residents and medical students: an analysis of 5-year follow-up data. Arch Intern Med 2000; 160:3107-3111.

4. Heald AE, Ransohoff DF. Needlestick injuries among resident physicians. J Gen Intern Med 1990; 5:389-393.

5. Osborn EH, Papadakis MA, Gerberding JL. Occupational exposures to body fluids among medical residents: a seven-year longitudinal study. Ann Intern Med 1999; 130:45-51.

6. Guo YL, Shiao J, Chuang YC, Huang KY. Needlestick and sharp injuries among health-care workers in Taiwan. Epidemiol Infect 1999; 122:259-265.

7. McGeer A, Simor AE, Low DE. Epidemiology of needlestick injuries in house officers. I Infect Dis 1990; 162:961-964.

8. Patterson JM, Novak $\mathrm{CB}$, Mackinnon SE, Ellis RA. Needlestick injuries among medical residents. Am J Infect Control 2003; 31:226-230.

9. Sohn S, Eagan J, Sepkowitz KA, Zuccotti G. Effect of implementing safetyengineered devices on percutaneous injury epidemiology. Infect Control Hosp Epidemiol 2004; 25:532-535.

\section{Incidence of Legionellosis in Hospitals Contaminated by Legionella pneumophila Other Than Serogroup 1}

TO THE EDITOR-In the late 1990s, following outbreaks of isolated cases or clusters of legionellosis in several Turin hospitals, the district attorney's office ordered all hospitals in its jurisdiction to monitor their water distribution systems. In response, Piedmont hospitals initiated surveillance and control measures to fully decontaminate their water distribution systems, regardless of whether legionellosis cases had occurred at the facility. Our department's involvement in evaluating the effectiveness of the decontamination interventions enabled us to gain a fairly complete picture of the situation at 36 hospitals, 35 of which were contaminated (61\% of samples tested positive for Legionella species, with bacterial loads ranging from $100-10,000 \mathrm{cfu} / \mathrm{L})$. Of the Legionella species isolated, Legionella pneumophila occurred most frequently; among the most common circulating serogroups were serogroup 6 (found at 15 hospitals), serogroup 3 (found at 12 hospitals), and serogroup 1 (found at 11 hospitals). Other species isolated (Legionella micdadei, Legionella steigerwaltii, and other unidentified strains) were consistently associated with L. pneumophila.

Concerned about legal action the magistrate's office could take against them if there was Legionella contamination in their water distribution systems, the hospital administrations initiated disinfection procedures recommended by guidelines (chlorination and thermal shock) or alternative methods (eg, disinfection with peracetic acid, ${ }^{2}$ ozone, and/or hydrogen peroxide), independently of the observed bacterial load or the presence of legionellosis cases. In the end, huge costs were incurred to obtain an effect that was as questionable as it was transient.

In this context, we invited the hospital administrations to participate in a 2-year project (2001-2002) that had the following objectives: to verify the incidence of nosocomial legionellosis in those hospitals contaminated with L. pneumophila other than serogroup 1 or with non-pneumophila species of Legionella, where only regular ordinary maintenance without decontamination measures would be carried out; and to monitor environmental contamination levels without decontamination measures.

In agreement with the medical departments of the hospitals participating in the project, the following protocol was set up. First, preliminary evaluation of environmental contamination was done. No environmental decontamination intervention was initiated at the central or peripheral parts of the water distribution system. Hospitals performed normal maintenance, defined as systematic interventions at distal outlets, instant hot water heaters, tanks (where present), air conditioning units, and cooling towers. There was semiannual monitoring of the water distribution system to identify spontaneous colonization. Also, hospitals performed active surveillance for Legionella infection for all cases of nosocomial pneumonia identified by clinical, radiological, and laboratory criteria. ${ }^{3}$ If a patient had pneumonia with onset at least 2 days after admission to the hospital, urine specimens were collected and examined for Legionella antigen; if the results were negative, the test was repeated 5-7 days later. Testing was performed using the Biotest Legionella Urine Antigen EIA

TABlE. Distribution of Pneumonia Cases at the 20 Study Hospitals, by Patient Age

\begin{tabular}{lc}
\hline Age, $y$ & No. $(\%)$ of cases \\
\hline$<40$ & $17(5)$ \\
$40-59$ & $48(14)$ \\
$60-80$ & $159(45)$ \\
$>80$ & $128(36)$ \\
\hline
\end{tabular}

